Characterization and optimization of a thin direct electron detector for fast imaging applications

This content has been downloaded from IOPscience. Please scroll down to see the full text.

2017 JINST 12 C03047

(http://iopscience.iop.org/1748-0221/12/03/C03047)

View the table of contents for this issue, or go to the journal homepage for more

Download details:

IP Address: 131.169.224.86

This content was downloaded on 12/07/2017 at 08:29

Please note that terms and conditions apply.

You may also be interested in:

Production quality characterisation techniques of sensors and prototypes for the BELLE II Pixel Detector

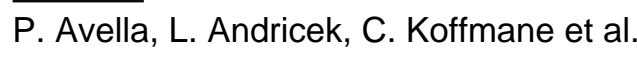

Single event imaging for electron microscopy using MAPS detectors

G McMullan, R Turchetta and A R Faruqi

Some aspects of the Pixel Vertex Detector (PXD) at Belle II

F Mueller

Finding an improved amorphous-silicon x-ray flat-panel detector configuration for the in-line geometry

M F Fast, A Teymurazyan, G Pang et al.

Principles and prospects of direct high resolution electron image acquisition with CMOSdetectors at low energies

A R Faruqi

A high frame rate, 16 million pixels, radiation hard CMOS sensor

N Guerrini, R Turchetta, G Van Hoften et al.

Direct Detectors for Electron Microscopy

R N Clough, G Moldovan and A I Kirkland

The Belle II DEPFET pixel detector

F Lütticke 


\title{
Characterization and optimization of a thin direct electron detector for fast imaging applications
}

\author{
I. Dourki, ${ }^{a, 1}$ F. Westermeier, ${ }^{a}$ F. Schopper, ${ }^{b}$ R.H. Richter, ${ }^{b}$ L. Andricek, ${ }^{b}$ J. Ninkovic, ${ }^{b}$ \\ J. Treis, ${ }^{b}$ C. Koffmane, ${ }^{b}$ A. Wassatsch, ${ }^{b}$ I. Peric, ${ }^{c}$ S.W. Epp ${ }^{a}$ and R.J.D. Miller ${ }^{a, d}$ \\ ${ }^{a}$ Max Planck Institute for the Structure and Dynamics of Matter, Center for Free Electron Laser Science, \\ Luruper Chaussee 149, 22761 Hamburg, Germany \\ ${ }^{b}$ Halbleiterlabor der Max-Planck-Gesellschaft, \\ Otto-Hahn-Ring 6, 81739 München, Germany \\ ${ }^{c}$ Karlsruhe Institute of Technology (KIT), Institute for Data Processing and Electronics (IPE), \\ 76021 Karlsruhe, Germany \\ ${ }^{d}$ Departments of Chemistry and Physics, University of Toronto, \\ 80 St. George Street, Toronto M5S 1H6, Canada \\ E-mail: ibrahym.dourki@mpsd.mpg.de
}

AbSTRACT: Direct electron detectors are increasingly used to explore the dynamics of macromolecules in real space and real time using transmission electron microscopy. The purpose of this work is to optimize the most suitable detector configuration of a thin silicon detector by Monte Carlo Simulations. Several simulations were performed to achieve an advanced detector geometry that reduces significantly the background signal due to backscattered electrons resulting in an enhanced imaging performance of the detector. Utilizing DEPFET (DEpleted P-channel Field Effect Transistor) technology and the novel ideas for the optimized detector geometry, a unique direct hit electron detector is currently being produced.

KEYWORDS: Detector modelling and simulations I (interaction of radiation with matter, interaction of photons with matter, interaction of hadrons with matter, etc); Solid state detectors

\footnotetext{
${ }^{1}$ Corresponding author.
} 


\section{Contents}

1 Introduction 1

2 Detector characteristics 2

3 Simulation results $\quad 2$

4 Modulation transfer function of the detector 5

5 Conclusions $\quad 6$

\section{Introduction}

Performing fast imaging experiments with low electron dose to explore dynamical processes occurring in real space and real time using transmission electron microscopy requires not only fast and bright electron sources but also high performance 2D detectors. Consequently, a crucial advance in the field of fast imaging experiments depends on the development of high speed pixel detectors with significantly improved detector performance. Direct electron detectors have become increasingly attractive to perform electron imaging experiments [1,2], as they potentially allow for a higher signal to noise ratio at a given electron dose.

We are currently developing a direct-electron-hit silicon detector operating with a maximum frame rate of about $80 \mathrm{kHz}$ named Edet80. It is based on DEPFET (DEpleted P-channel Field Effect Transistor) technology [3]. The DEPFET concept and thinning technology provide the possibility to vary the sensor thickness over a wide range [4] according to the needs of the experiment. In addition, its low power consumption allows to build thin detectors without extra material for cooling and mechanical support. A particle of sufficient high energy loses only a fraction of its energy in a thin sensor which has the advantages of minimizing the effect of multiple electron scattering and charge sharing between neighboring pixels and leads thus to an improved image resolution. Moreover, for a given charge handling capability the number of detected events in each pixel is enhanced as the amount of charge generated per event decreases. Due to its good noise performance even at high frame rates and its internal amplification, the DEPFET is able to detect small signals, which allows the detection of single electron hits with high probability. These features make DEPFET pixel detector a promising candidate to explore fast dynamics occurring in biological samples on sub-millisecond timescale (e.g. protein folding dynamics) using transmission electron microscopy and in particular when the sample being investigated is very thin, consists of low atomic number elements and must be irradiated with limited electron dose to avoid radiation damage of the sample which results in few electron scattering events and low contrast images.

Monte Carlo Simulations can be a powerful tool for the optimization of detectors and exploring the significance of several physical effects limiting the detector performance. An efficient particle 
transport framework like Geant4 [5] offers the possibility to construct geometric models of detectors, experimental setups and simulate the interaction of particles with the material of the detector as well as to optimize the most suitable detector and experimental setup configurations. Here, we attempt to optimize the detector geometry and show design ideas to minimize backscattered electrons from the detector housing. We achieve a detector design showing improved imaging performance very close to a detector without the background signal generated by backscattered electrons.

\section{Detector characteristics}

Edet 80 will be a Megapixel detector arranged in four quadrants with a $512 \times 512$ pixel matrix each. The pixel size is $60 \mu \mathrm{m} \times 60 \mu \mathrm{m}$. To combine a large charge handling capability with excellent detection efficiency, the sensitive area is thinned down to $50 \mu \mathrm{m}$. In such a thin sensor, high energy electrons deposit only a small fraction of their energy. To increase the charge handling capability further, a non-linear signal storage and compression stage is being implemented. The sensor matrix is read out in rolling-shutter mode with column parallel amplification and digitization. To increase the readout speed, the rolling shutter is implemented four-fold, thus requiring 2048 readout chains per quadrant. The readout speed is thus increased by a factor of four. As in the Belle II pixel detector [6], one sensor row can be read out in $100 \mathrm{~ns}$ and thus $100 \mathrm{~ns} \times 128$ results in total readout of $12.8 \mu$ s for an entire frame which corresponds to $78.1 \mathrm{kHz}$ frame rate. This speed is sufficient to capture a large range of dynamics in chemistry and biology in real time.

\section{Simulation results}

Geant4 Monte Carlo simulation is an useful tool to develop and optimize new detector concepts. It allows to design, predict and improve the detector performance. We start our investigation by calculating the signal (the number of electron hole pairs) generated in a sensitive layer of silicon of increasing thicknesses and at different energies of the incident electrons. Figure 1 (left) shows that the number of electron hole pairs (e-h pairs) generated in silicon layer depends on the thickness and the energy of the impinging electrons. As the material thickness increases, an electron traveling through the material loses a higher fraction of its energy. For instance, in $50 \mu \mathrm{m}$ and $500 \mu \mathrm{m}$ thick silicon layers a primary electron of $300 \mathrm{keV}$ generates approximately an average of $7780 \mathrm{e}$-h pairs and 76500 e-h pairs respectively. This energy loss decreases with increasing electron energy for the considered energy range. The energy loss distribution of electrons impinging on a thin silicon layer can be well described by a Landau distribution [7] which is an asymmetric distribution with a long tail characterized by the mean value (mv) and the most probable value (mpv). The signal distribution in a $50 \mu \mathrm{m}$ silicon layer irradiated with a $300 \mathrm{keV}$ electron beam is shown in figure 2 and it obeys Landau distribution. The most probable value and the mean value are $4850 \mathrm{e}-\mathrm{h}$ pairs and $7780 \mathrm{e}-\mathrm{h}$ pairs respectively. The mean energy of electrons after traversing $50 \mu \mathrm{m}$ silicon layer shown in the inset of figure 2 is about $270 \mathrm{keV}$. This means that only $10 \%$ of the incident electron energy is absorbed. As only a given amount of charge can be stored in the internal gate of a DEPFET pixel detector, a thin sensor is essential to enhance the charge handling capability.

In addition, having such a thin silicon sensitive layer reduces the contribution of multiple electron scattering to the point spread function and improves thus the spatial resolution of the 

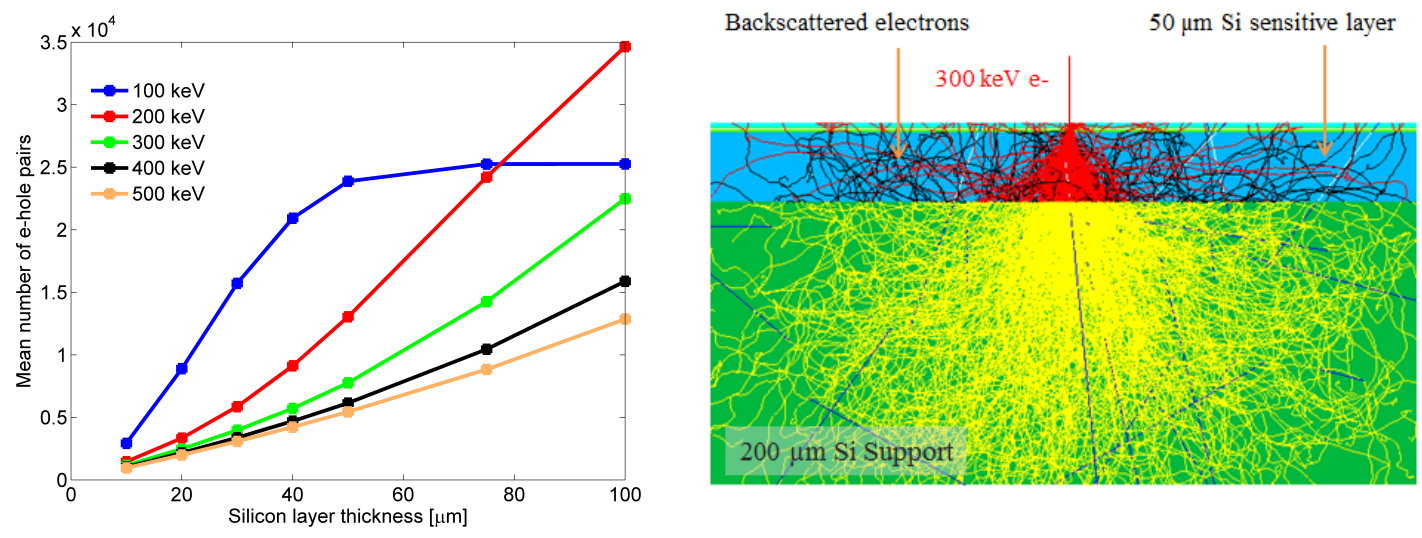

Figure 1. Left: mean number of electron hole pairs per primary electron generated in a silicon layer of increasing thickness and for electron energies from 100 to $500 \mathrm{keV}$. Right: Geant 4 simulation of the tracks of 500 electrons of $300 \mathrm{keV}$ in a detector consisting of different layers. Blue is a silicon sensitive layer of 50 $\mu \mathrm{m}$ thickness and green is a $200 \mu \mathrm{m}$ thick silicon support layer. Black trajectories represent backscattered and secondary electrons emitted from the support layer which travel back into the sensitive layer. Blue trajectories are X-rays in the support layer, white trajectories are X-rays in the sensitive layer and yellow tracks are electrons in the support layer.

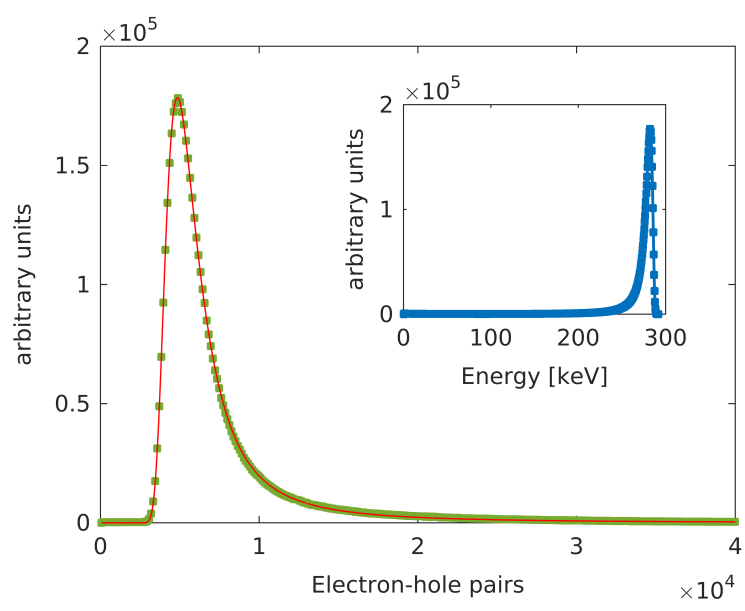

Figure 2. Distribution of the e-h pairs generated in a $50 \mu \mathrm{m}$ thick silicon layer per $300 \mathrm{keV}$ primary electron (green squares). The solid red line is a fit of a Landau distribution to the data. The inset shows the energy spectrum of electrons after traversing the silicon layer.

detector. However, the large fraction of high energy electrons traversing a thin silicon sensitive layer leads to a background signal produced by backscattered electrons (see figure 1 (right)) from the detector housing and supporting substrate which degrades the detector performance. Therefore, a simulation study of different detector geometries has been carried out trying to reduce this unwanted signal. One way to reduce the signal generated by backscattered electrons is to prevent these electrons from reaching the sensitive layer by introducing a vacuum gap between the sensitive layer and the supporting substrate. An example of a geometrical setup and the resulting signal with homogeneous illumination of the sensitive area is shown in figure 3 . 

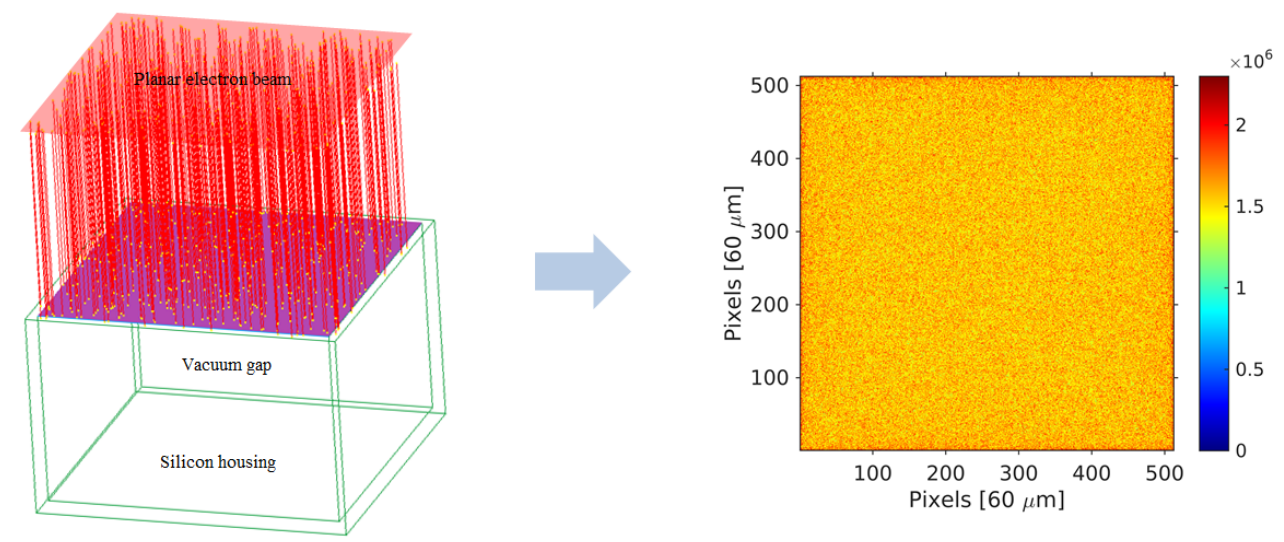

Figure 3. Left: example of a simulation setup of the detector including a silicon housing and a vacuum gap. Right: signal distribution in the sensitive area.

From the signal distribution shown in figure 3 (right), the mean signal is calculated to find an optimized vacuum gap. The results presented in figure 4 show that without vacuum gap the detected signal is increased by about $58 \%$ with respect to the signal without any backscattering. This additional signal reduces the signal to noise ratio and degrades thus the detector imaging performance. The introduction of a vacuum gap reduces the signal caused by backscattered electrons. For instance, using a vacuum gap of $20 \mathrm{~mm}$ reduces the unwanted signal from $58 \%$ to $16 \%$. The number of backscattered electrons increases with increasing atomic number of the detector housing material. When the atomic number of the material decreases, the electron reflection coefficient decreases which in turn reduces the contribution of the backscattered electrons to the signal. Thus, the background signal can be further reduced by coating the detector housing with low atomic number materials such as carbon and beryllium. Figure 4 shows that with $20 \mathrm{~mm}$ vacuum gap and $200 \mu \mathrm{m}$ carbon coating the background signal is reduced to 5.4\%.

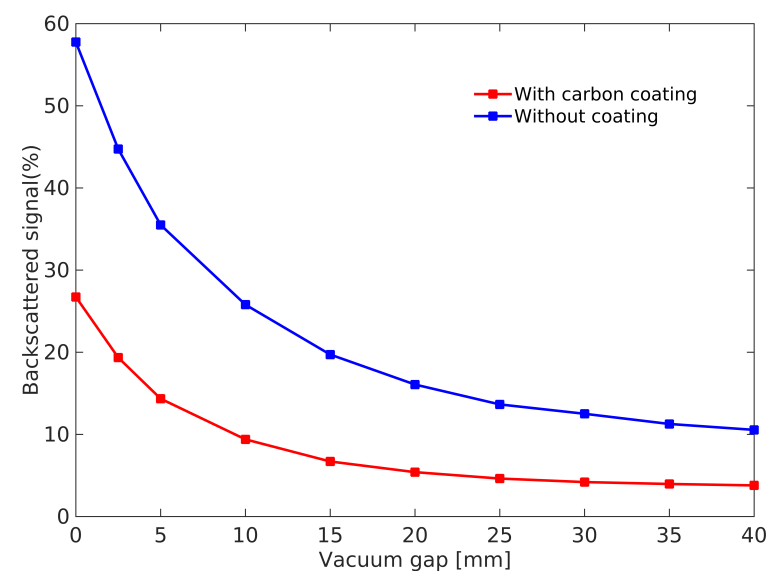

Figure 4. Additional signal due to backscattered electrons as a function of distance between sensitive layer and detector backplane. A comparison between coated and uncoated detector geometry is shown. Coating the detector geometry with carbon reduces the additional signal generated in the sensitive layer. 


\section{Modulation transfer function of the detector}

The spatial resolution of imaging detectors is usually expressed in terms of the modulation transfer function (MTF) specified in ISO standard 12233 [8]. The MTF measures the ability of a detector to preserve signal contrast as a function of spatial frequency. It can be calculated using, for example, the slanted edge method [9-11] which is widely used, easily implemented and gives accurate results. This method is realized by imaging a sharp edge slightly tilted with respect to the pixel matrix to increase the sampling rate of the edge spread function (ESF). The differentiation of the ESF is the Line Spread Function (LSF) which gives the MTF by performing a Fourier transformation [12]. The image of such a slanted edge target obtained by Monte Carlo simulation is shown in figure 5 . It shows the number of e-h pairs created in a $50 \mu \mathrm{m}$ thick silicon sensor by a $300 \mathrm{keV}$ electron beam with a fluence of 205 electrons per pixel. The pixel size is $60 \times 60 \mu \mathrm{m}^{2}$, and no additional readout noise has been assumed for MTF calculation. The comparison between MTFs of different detector geometries of $60 \mu \mathrm{m}$ pixel size is shown in figure 6.

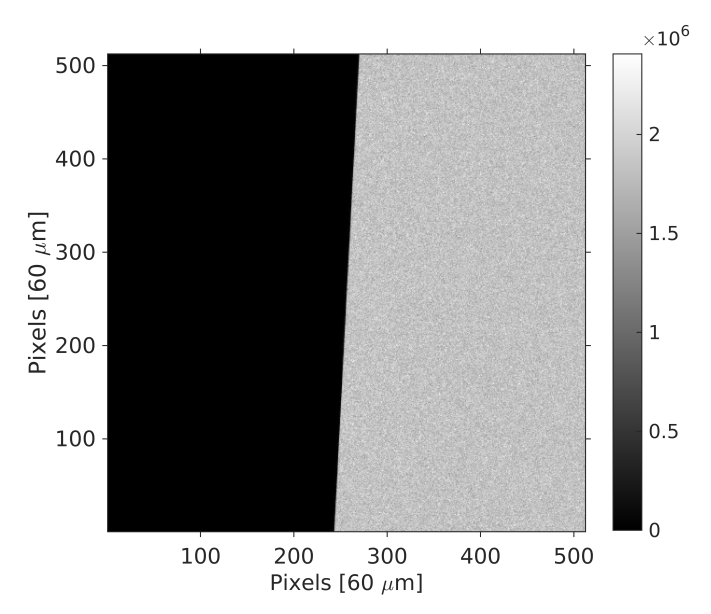

Figure 5. Simulated slanted edge target. The color code refers to the number of e-h pairs created in a $50 \mu \mathrm{m}$ thick silicon sensor by a $300 \mathrm{keV}$ electron beam with a fluence of 205 electrons per pixel.

In the case of a sensor directly supported by a thick silicon substrate (as shown in figure 1 (right)), the signal generated and detected in the sensor is the sum of two adjacent energy deposits, one from the incoming electrons and the second one from the backscattered electrons. As demonstrated (see figure 4), by using a silicon support of $300 \mu \mathrm{m}$ thickness, the backscattering contribution adds about $60 \%$ intensity to the signal detected in the sensor. This degrades strongly the MTF and makes it fall rapidly with increasing spatial frequency. In contrast, a detector design including a vacuum gap and a detector housing coated with carbon shows an imaging performance in terms of spatial resolution with a MTF very close to the MTF of a detector without any backscattering (see figure 6). Thinning the silicon sensor and reducing the amount of backscattered electrons lead to an enhanced MTF. This MTF improvement by thinning the sensor was experimentally demonstrated by McMullan et al. [13]. Thinning the sensitive layer even further (e.g. $30 \mu \mathrm{m})$ results in less charge spreading and a further remarkable improvement of the MTF. 


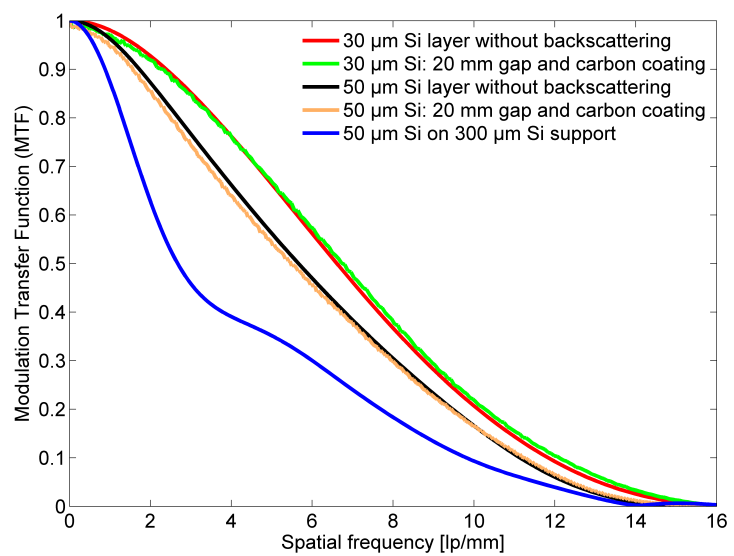

Figure 6. Comparison of MTFs for five different detector geometries irradiated with $300 \mathrm{keV}$ electrons. Introducing a vacuum gap and coating the detector housing improve considerably the MTF of the detector. The MTFs are normalized to 1 at a spatial frequency of 0 and decrease as the spatial frequency increases.

\section{Conclusions}

A thin sensitive layer improves both the charge handling capability and the spatial resolution of an electron detector based on DEPFET technology. However, due to the small energy loss of the detected electrons the signal created by backscattered electrons degrades the detector performance. This simulation study demonstrates that the issue of additional signal generated by backscattered electrons can be overcome. The fraction of backscattered electrons depends on the atomic number of the detector housing material and can be significantly minimized by introducing a vacuum gap and coating the detector housing by low atomic number materials such as carbon which results in an improved imaging performance of the detector. The advanced detector design developed in this study can be useful for future detector developments. Based on these detector simulation results, the mechanical housing of a novel electron detector is now being manufactured.

\section{References}

[1] A.R Faruqi and R. Henderson, Electronic detectors for electron microscopy, Curr. Opin. Struct. Biol. 17 (2007) 549.

[2] N. Xuong et al., Future Directions for Camera Systems in Electron Microscopy, Method Cell Biol. 79 (2007) 721.

[3] J. Kemmer and G. Lutz, New detector concepts, Nucl. Instrum. Meth. A 253 (1987) 365.

[4] S. Rummel, The power supply system for the DEPFET pixel detector at BELLE II, Nucl. Instrum. Meth. A 699 (2013) 51.

[5] GEANT4 collaboration, S. Agostinelli et al., GEANT4: A Simulation toolkit, Nucl. Instrum. Meth. A 506 (2003) 250.

[6] M. Schnell, The Belle II Pixel Detector for the SuperKEKB Flavour Factory, proceeding of the $23^{\text {rd }}$ International Workshop on Vertex Detectors, Macha Lake, The Czech Republic, 15-19 September 2014. 
[7] L. Landau, On the Energy of Fast Particels by Ioniziation, J. Phys. (USSR) 8 (1944) 201.

[8] ISO 12233, Photography - Electronic still picture cameras - Resolution measurements, ISO, (1998).

[9] M. Estribeau and P. Magnan, Fast MTF measurement of CMOS imagers using ISO 12333 slanted-edge methodology, in Detectors and Associated Signal Processing, Proc. SPIE 5251 (2004) 243.

[10] A. Walter and S. Lashansky, An improved method for calculating the MTF of an optical system, in Sensors, Cameras, and Systems for Scientific/Industrial Applications VII, Proc. SPIE 6068 (2006) $60680 \mathrm{~S}$.

[11] S.N. Lashansky, S. Mansbach, M.J. Berger, T. Karasik and M. Bin-Nun, Edge response revisited, in Infrared Imaging Systems: Design, Analysis, Modeling, and Testing XIX, Proc. SPIE 6941 (2008) $69410 Z$.

[12] Y. Kao, M. Albert, A. Carton, H. Bosmans and A.D.A. Maidment, A software tool for measurement of the modulation transfer function, Proc. SPIE 5745 (2005) 1199.

[13] G. McMullan et al., Experimental observation of the improvement in MTF from backthinning a CMOS direct electron detector, Ultramicroscopy 109 (2009) 1144. 